\title{
An Efficient Study of Scheduling Algorithms with Friedman Test in WiMAX Networks
}

\author{
Mohamed-El-Amine Brahmia \\ LUSINE-CESI, Strasbourg \\ 2 Allée des Foulons - 67380 Lingolsheim, France \\ Tel: +33-3-88-10-38-01Ｅ-mail: abrahmia@cesi.fr \\ Abdusy Syarif, Abdelhafid Abouaissa, Lhassane Idoumghar, Pascal Lorenz \\ University of Haute Alsace \\ 34 Rue de Grillenbreit - 68008 Colmar, France \\ Tel: +33-6-32-63-02-04 E-mail: \{abdusy.syarif, abdelhafid.abouaissa, \\ lhassane.idoumghar, pascal.lorenz\}@uha.fr
}

Received: November 7, 2014 Accepted: November 22, 2014 Published: December 27, 2014

DOI: 10.5296/npa.v6i4.6582

URL: http://dx.doi.org/10.5296/npa.v6i4.6582

\begin{abstract}
In this paper, we evaluate and compare our scheduling strategy for various IPTV services traffic over 802.16j networks with several scheduling algorithms such as Strict Priority algorithm (SP), Weighted Round Robin (WRR), and Modified Dynamic Weighted Round Robin (MWRR). The proposed scheme adapts dynamically the scheduler operation to according queue load and QoS constraints. In particular, the proposed mechanism gives more priority to HD-TV and SD-TV traffics by using two schedulers. The proposed scheduling algorithm has been simulated using the QualNet network simulator. The Friedman test has been used to address some significant issues of the analysis to compare the proposed scheme with the others scheme. The experimental result and analysis show that the proposed scheduler schemes outperform the traditional scheduling techniques for rtPS traffic that allows ensuring QoS requirements for IPTV application.
\end{abstract}

Keywords: IEEE 802.16j, WiMAX, Scheduling, Friedman test. 


\section{Introduction}

In WiMAX standard, the tools to set up a network of high-speed data have been described in [1], but it depends on the operators which implement their own mechanisms for Quality of Service (QoS) management purpose. It is a very challenge topic to optimize the mechanism of QoS management that we are interested.

A WiMAX technology is an emerging wireless technology for deploying broadband Wireless Metropolitan Area Network (WMAN). It is a very promising Broadband Wireless Access (BWA) technology which able to transmit different service types. This technology could has different constraints such as traffic rate, maximum latency, and jitter tolerance. The IEEE 802.16 Medium Access Control (MAC) standard specifies five types of quality of service (QoS) classes: Unsolicited Grant Service (UGS), real-time Polling Service (rtPS), extended real-time Polling Service (ertPS), non real-time Polling Service (nrtPS), and Best Effort (BE). However, the IEEE 802.16 standard does not specify the scheduling algorithm to be used. The communication operators should choice among many existing scheduling techniques. And also, they could propose their own scheduling algorithms.

We present a new mechanism to differentiate services applied to WiMAX network at the MAC layer. This proposed scheduling mechanism is designed for IPTV application which is part of real-time Polling Service (rtPS) service class. We assumed that each video stream could be encoded into four categories (HD, SD, Web TV and Mobile TV). The proposed algorithm is weighted based called Weighted Round Robin (WRR) scheduling technology. The aim of this proposed mechanism is to adapt dynamically in relation to the management of queues and constraints of each class of service. Furthermore, we evaluate the performance of the proposed scheduling algorithm with simulation and statistical analysis.

This paper presents a Friedman test [15] which compares the performances of proposed algorithm with others algorithm. The Friedman test is one of the nonparametric tests of multiple group measures. In other words, it can be used to approve the null hypothesis that the multiple group measures have the same variance to a certain required level of significance.

The rest of this paper is organized as follows. Section 2 reviews related work. Section 3 discusses the proposed work. Simulation and results are discussed in Section 4 and conclusion of the paper is presented in Section 5.

\section{Related Work}

\subsection{Scheduling in WiMAX Networks}

In IEEE 802.16 standard, MAC layer is connection oriented and base station assigned by a unique connection identifier (CID) for both transmissions, Up-Link (UL) and Down-Link (DL) [2]. However, at Convergence Sub-layer (CS), data streams are classified into different connections with different scheduling services. Therefore, it is expected that several schedulers are implemented to meet heterogeneous demands of users. There are two models 
of resource allocation and scheduling for SSs in 802.16j standard [3], centralized and distributed scheduling. The first model, resources allocation for all nodes is ensured by MR-BS. While the second mode, resources allocation and bandwidth are determined by MR-BS and RSs in non-transparent relay. Knowing that a non-transparent relay can support both modes, and then we are interesting in the centralized method.

Furthermore, as far as we know, there is no scheduling technique specified in 802.16 standard. Each operator could develop its own technology to provide its capacity and applications respectively.

\subsubsection{The different types of services}

The standard in [1] specified five types of services to serve connections at MAC layer. Each type of service is an application type with a traffic profile particular. These services are:

- Unsolicited Grant Service (UGS): This type of service provides a service flow which the transmission system automatically and provides a defined number of timeslots periodically and fixed packet size which is used by a particular receiver. UGS is frequently used to provide services that involve a constant bit rate (CBR), for example audio streaming application or leased line circuit emulation.

- Extended Real-Time Polling Service (ertPS): This service allows real-time service flows that periodically generate variable-sized data packets, such as VoIP application.

- Real-Time Polling Service (RTPS): This type of service corresponds to real-time data streams comprising variable-sized data packets which are released periodically.

- Non-Real-Time Polling Service (nrtPS): This type of service corresponds to delay-tolerant data streams comprising variable-sized data packets for which a minimum data rate is needed, such as file transfer (FTP) with a guaranteed minimum throughput.

- Best Effort (BE): This type of service corresponds to data streams for which no minimum service level is necessary and therefore could be treated on a space-available basis, for example HTTP application.

\subsubsection{Scheduling Algorithm}

Scheduling algorithm is the most important aspect of resources efficient in WiMAX networks. A scheduling algorithm should take into account when considering a quality of service (QoS) constraints for all classes of service. There are two types of scheduling algorithms. The first type of schedulers known as RR, WWR, SP, etc.. The second type of scheduler is proposed specifically for WiMAX networks [4].

Among known scheduling techniques which we will compare later with the proposed scheduler, we chose two scheduling algorithms as following:

- Weighted Round Robin (WRR): This scheduling algorithm is designed for a better management services with different QoS requirements. It assigns a weight to each 
static queue, and then bandwidth is allocated based on these static weights [5]. In addition, queues with higher priority have more connections (bandwidth) than queues with lower weight. WRR ensures all service classes have access to bandwidth network, which avoids the problem of resource starvation in low priority queue [6].

- Strict Priority (SP): This scheduling technique is simple. It begins to serve queues with high priority until empty, and then it goes to the next queue with highest priority. By using this scheduler, queues with low priority may encounter the problem of resource starvation [7].

The authors of [8] proposed a modified version of the dynamic scheduler WRR called MDWRR (Modified Dynamic Weighted Round Robin). The objective of their proposal is to ensure the processing time for real-time applications, while efficiently managing non-real time traffic. The idea of their proposed scheduler is a variant of the algorithm DWRR (Dynamic Weighted Round Robin) using a threshold to avoid packet loss of non-real time traffic. Although MDWRR algorithm is more complex than the WRR and DWRR technique, it takes into account the priority of services and reduces the required size of buffer. Unlike the MDWRR algorithm, our proposed mechanism takes into account the QoS constraints of all service classes and load queues.

In [6] the authors presented a scheduling algorithm for WiMAX networks. Their proposed algorithm based on WRR and appointed to MWRR (Modified Weighted Round Robin). This algorithm designed with to reduce the average end-to-end delay and to improve the average throughput. The authors compared the MWRR algorithm with known scheduling techniques (WRR, SP and WFQ). The results show that the algorithm MWRR tends to be reliable, especially for classes of service low priority which avoids the problems of the WRR technique that causes unnecessary delays. In the performance evaluation section, we compare our proposed scheduling mechanism with MWRR algorithm.

The authors of [7] conducted a study on scheduling algorithms such as WFQ, RR, WRR and SP in WiMAX network. They analysed and evaluated the performance of each scheduler by comparing the behaviour of each algorithm with respect to different service classes. Their simulation results showed that the SP scheduler has the best throughput and the minimum delay for high priority service class. However, it has caused the resource starvation problem for $\mathrm{BE}$ and nrtPS classes. The results indicated that the SP algorithm as the average end-to-end is important for rtPS class. However, the scheduler RR had good results for classes of service with low priority, ignoring the constraints of QoS classes of high priority. Furthermore, algorithms WFQ and WRR can monitor the performance of each service class by assigning different weights to each queue.

The authors in [21] have investigated the distributed video scheduling with delayed control information (DCI). They translated the scheduling problem into a stochastic optimization rather than a convex optimization problem. In [22], they proposed a network algorithm that supervises the IPTV access network and determines which type of wireless technology the clients should connect with when using multiband devices, depending on the requirements of the IPTV client device, the available networks, and some network parameters 
(such as the number of loss packets and packet delay), to provide the maximum Quqlity of Experience (QoE) to the client. The authors in [23] proposed a novel IPTV service based on the concept of content-zapping, in contrast to traditional channel-zapping for the distribution of personalized multimedia contents over IP networks, where each customer system obtains a multimedia streaming that is automatically arranged by the system based on the user selections and the user will only associate with the system by requesting a content change or marking a content as favorite.

In the next section, we present the proposed scheduling algorithm which is based on the WRR technique. The performance of the proposed scheduler will be studied by using the QualNet simulator. Then, we perform analysis to compare the performance of proposed algorithm and techniques WRR, SP and MWRR [6].

\subsection{The Nonparametric Test}

Statistical tests frequently are in the form of parametric tests which by definition test an hypothesis about the parameters of the probability distribution function (pdf) of a sample of data. Contrarily, a non parametric test doesn't need any formulation of the parameters of the pdf. The test used in this present work is the Friedman test for the analysis of the variance (ANOVA) in order to perform the comparison of quality of services.

The Friedman test is also a non parametric test of variance performed on ranks, but in contrast to the Kruskal Wallis test it does not need the hypothesis that the samples are independent. All parametric ANOVA's have to assume that the data is on the interval or ratio scales and is distributed normally. In the same way that the Mann-Whitney test gives a non-parametric alternative to the t-test, therefore the Kruskal-Wallis test gives the alternative non-parametric procedure.

The Kruskal-Wallis test does not require equal sample sizes but it will order about which post hoc tests can be used. The data does not need to be in matched groups but if it is, there is a further test, the Friedman test that can be used instead.

The Kruskal-Wallis test relates to the Friedman test. Therefore essentially the Friedman test is used in case you want to use the same sample of subjects or cases and evaluate them at three or more points in time or under differing conditions.

In order to achieve a better performance comparison between the scheduling algorithms, we performed a statistical analysis based on the Friedman test. [16], [17], [18], [19], [20] have performed statistical analysis by using the Friedman test for their work.

The authors in [18], they used two tests, the Kruskal-Wallis and the Friedman test, to address two significant issues of the analysis of long time series in their work. In order to address issue of overcoming noise uncertainty by exploiting antenna correlation, in [19], the authors presented a Friedman test based spectrum sensing detector which compares the power of the received signals through antennas and consequently it needs no priori information of the noise and the primary signal. In [17] and [20], the autors deal with some QoS in Wireless Sensor Network (WSN) and Wireless Mesh Network (WMN). They used the Friedman test to 
compare their proposed deployment method with others algorithm such as Genetic Algorithm (GA) and Tabu Search (TS).

\section{Proposed Work}

\subsection{Adaptive scheduling with dynamic weight adjustment (AWRR)}

This section show the proposed scheduling algorithm which is based on weighted WRR scheduler. The proposed mechanism is based on two criteria, the load of each queue and the QoS constraints of each service class. The objective of this technique is to dynamically adjust the weight of each queue in relation to those two criteria above. This technique is called Adaptive Weighted Round Robin (AWRR) [9]. The weight management mechanism is provided by a system of equations which is based on the detection of a crossing certain threshold that anticipates congestion packet loss due to queue overflow. The AWRR algorithm allows providing service differentiation in a multi-files system to wait for relays network in case of centralized management.

Apart from management of all service classes, the proposed scheduler gives more priority to IPTV services that have more constraints, such as video stream with HD or SD quality. We assume in the proposed transmission process, we use four IPTV services (HD, SD, Web TV and Mobile TV) which are part of the rtPS class.

Moreover, Weighted Round Robin (WRR) is designed to manage different classes of service such as real-time application, interactive application, file transfer, etc. The weight is associated with each queue that is specifically dedicated to one of the service classes. Each queue is serviced in a round robin order according to its weight. The queue with the highest priority weight takes to process more packets [6], [10]. However, the proposed mechanism uses WRR scheduling technique for two reasons, first, it allows avoiding the problem of resource starvation for lower priority classes, and the second, it gives the QoS guarantee in high-priority classes.

The idea in this work is to use two schedulers in both case, at input and output sides. The scheduler at input side has two queues, first, High Priority $(H P)$ which contains flow of UGS, ertPS and rtPS classes. Otherwise, the flow rtPS is only for IPTV services encoded in HD or SD. The second queue called Low Priority $(L P)$ which contains flow of the rest of service classes, in this case rtPS web-TV, rtPS mobile-TV, nrtPS and BE. The scheduler at output side is the main scheduler that can control and manage all service classes (UGS, ertPS, rtPS, nrtPS and BE).

As mentioned above, the role of input scheduler is to support video streams which have more constraints in terms of bandwidth and latency. Using this scheduler does not have affect with the processing time, as the data packets have a removal time before they are processed by the main scheduler. 


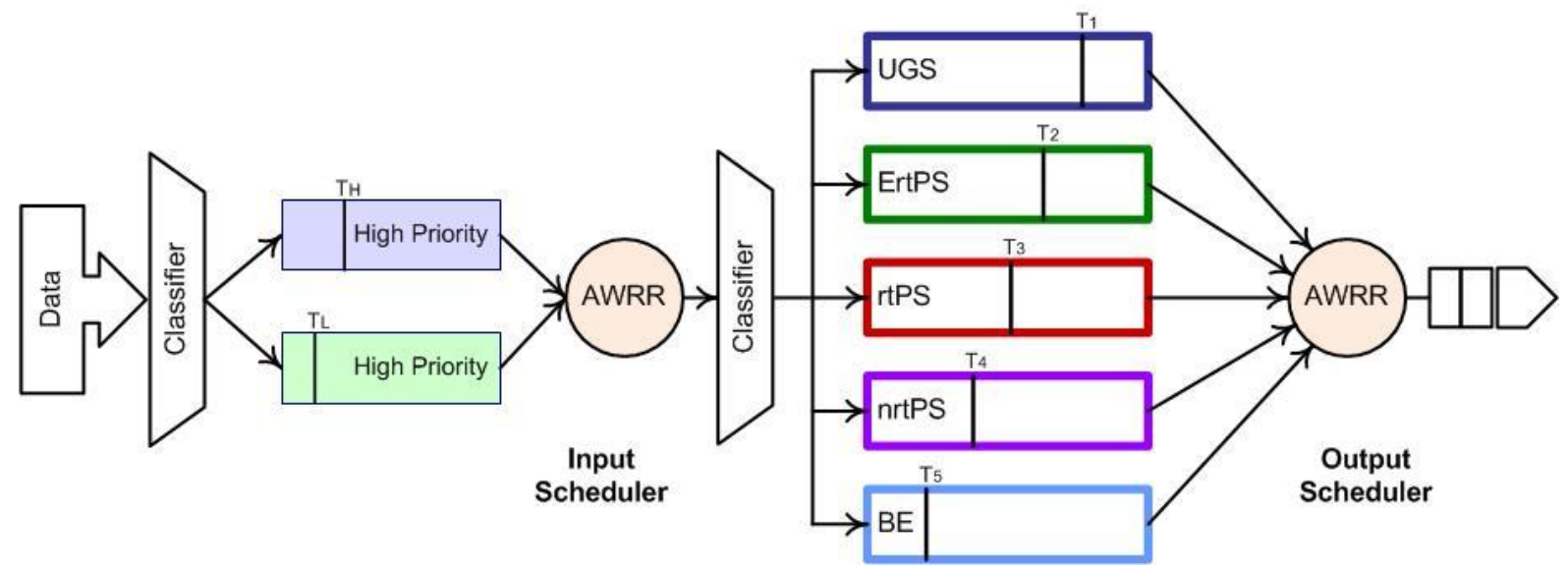

Figure 1. The architecture of AWRR scheduler.

We recall from our previous work [9], Figure 1 shows the overall architecture of proposed scheduler with specifying different queues. The proposed algorithm applies AWRR on both schedulers (input and output) to adjust the weight of each service class. It also assigns a congestion threshold at each queue which is used to trigger the dynamic weight adjustment in case of overflow. Thus, to meet the QoS constraints and in order to give higher priority to real-time applications, it assumed that the thresholds of the high priority queues are above the threshold of low-priority queues.

The following section presents the detail of two schedulers, in particular, the adjustment of queue weight.

\subsubsection{The Input Scheduler}

Input scheduler has two queues, high priority queue $(H P)$ and low priority queue $(L P)$. The classification of data is divided in a manner of HD-TV and SD-TV services which is belong to HP. Web-TV and Mobile-TV services are classified in $L P$.

The main feature of the input scheduler is to control the weight of each line according to traffic load and buffer size. The weight adjustment can favor the data processing belonging to HP while guaranteeing a minimum processing time for service classes belonging to $L P$.

\subsubsection{Queue Weight Adjustment (Input Scheduler)}

The weight adjustment of two queues takes into account the load and the priority each queue. For this purpose, two thresholds $\left(T_{H}\right.$ and $\left.T_{L}\right)$ are used as seen at Figure 1. Every threshold represents the number of packet occupying the queue. The weight adjustment takes into account the basic constraint: $W_{H}+W_{L}=1$. Knowing that the weight of two queues is defined by following equations:

$$
\begin{gathered}
W_{H}=W_{H i}+\frac{N P_{H}-T_{H}}{N P_{H}} \\
W_{L}=1-W_{H}
\end{gathered}
$$


Where $W_{H}$ is the weight of high priority queue, $W_{L}$ is the weight of low-priority queue, $T_{H}$ refers to the threshold of high priority queue, and $N P_{H}$ refers to the number of packet occupying the $H P$.

The threshold $\left(T_{H}\right)$ is used to trigger the weight adjustment the number of packet occupying the queue exceeds the threshold. $W_{H i}$ is the weight initial of $H P$ which allows the AWRR scheduler to calculate $W_{H}$ at the first weight adjustment.

\subsubsection{Weight Calculation Algorithm}

The algorithm 1 calculates the weight of each queue based on the packet amount in each queue. In this algorithm, $T_{L}$ represents the threshold of low-priority queue and $N P_{L}$ is the number of packet occupying the queue.

Algorithm 1 Calculation of weight (input scheduler)

1. $W_{H i}=\alpha$

2. $(\alpha<1)$

3. $W_{L}=1-W_{H i}$ / * Initial Conditions * /

4. while (Queues are not empty) do

5. if $N P_{H} \geqslant T_{H}$ or $N P_{L} \geqslant T_{L}$ then

6. $\quad W_{H}=W_{H i}+\frac{N P_{H}-T_{H}}{N P_{H}}$

7. $\quad W_{L}=1-W_{H}$

8. else

9. Initial_Conditions

\subsubsection{The Output Scheduler}

The output scheduler is used to meet the requirements in terms of quality of service. It manages the flow of all service classes by using AWRR. The architecture of output scheduler has a queue for each service class. Each of this service class has a weight and a threshold to detect the certain level of congestion. The weight adjustment must meet the requirements of each service class, as it must avoid problem of resource starvation for low-priority queues. For this reason, the proposed method relies on a system of equations in order to adjust the weights dynamically. Knowing that each weight as a linear equation, then the equation takes into account the number of packets occupying a queue, the threshold assigned and the constraints of service class.

\subsubsection{Queue Weight Adjustment (Output Scheduler)}

The adjustment of queue weight is a very important function for the proposed mechanism in order to improve the management of queue mechanism. This function provides the weight adjustment of the five queues dynamically to take into account the following constraints: 


$$
\begin{gathered}
W_{i}>0 \\
\sum_{i=1}^{m} W_{i}=1
\end{gathered}
$$

Where $m$ represents the number of queue and $W_{i}$ is the weight of the $i-t h$ row. However, we assume that:

$$
\begin{gathered}
W_{i}=f_{i}(k)=a_{i} k+b_{i} \\
a_{i}=\frac{N P_{i}}{T_{i}}
\end{gathered}
$$

Including $N P_{i}$ is the number of packet occupying the $i-t h$ row and $T_{i}$ is the threshold for the queue. However, $b_{i}$ is a predefined constant that can support high-priority classes.

$$
\text { (4) } \Rightarrow \sum_{i=1}^{m} f_{i}(k)=1 \Rightarrow \sum_{i=1}^{m}\left(a_{i} k+b_{i}\right)=1
$$

Once variable $k$ is calculated, we can calculate the weight of each queue (service class). Moreover, the values of two constants are arbitrarily prefixed in order to increase the weight of higher priority classes. In this case, we have five queues, so we assume that $\left(b_{1}=\alpha_{1}\right.$ for $U S G, b_{2}=\alpha_{2}$ for ErtPS, $b_{3}=\alpha_{3}$ for rtPS, $b_{4}=\alpha_{4}$ for nrtPS and $b_{5}=\alpha_{5}$ for BE).

\subsubsection{Weight Calculation Algorithm}

The weight assigned to each queue is always positive value to ensure a minimum processing time of low-priority classes. The proposed mechanism supports to high priority classes using constant $b_{i}$ and threshold $T_{i}$. The calculation algorithm provides three weights as we described above.

Algorithm 2 Weight Calculation (Output Scheduler)

1. $W_{i}>0 ; \sum_{i=1}^{m} W_{i}=1$

2. / * Initial Conditions $*$ /

3. $b_{1}=\alpha_{1} ; b_{2}=\alpha_{2} ; b_{3}=\alpha_{3} ; b_{4}=\alpha_{4} ; b_{5}=\alpha_{5}$

4. while (Queues are not empty) do

5. for $i=1$ to 5 do

6. if $N P_{i} \geqslant T_{i}$ then

7. Weights_Ajustment else

8. Initial_Conditions 


\section{Performance Evaluation}

In order to highlight the proposed scheduling mechanism, we used QualNet [11] simulator to implement the proposed algorithm on WIMAX network. We compare AWRR to WRR, SP and MWRR [6] to show the effectiveness of the proposal. We chose MWRR because it also based on WRR and designed for WiMAX networks.

\subsection{Parameters Simulation}

In this simulation, we used $802.16 \mathrm{e}$ since $802.16 \mathrm{j}$ version doesn't implement a scheduler. This choice does not influence the performance evaluation of the proposed algorithm because it is a centralized solution, it means that base station which handles the scheduling.

In scenario simulation, we consider that the topology has a WiMAX cell consisting of 1 BS and 50 SSs. At the application layer, we use CBR (Constant Bit Rate) traffic generator and specifying the service class for each $\mathrm{CBR}$ connection using the precedence field (UGS=7, ertPS $=4, \operatorname{rtPS}=3$, nrtPS=2, and $\mathrm{BE}=0$ ). In addition, we are particularly interested in rtPS class by changing the number and the quality of IPTV channels in order to study the impact of proposed input scheduler.

Table 1 shows the simulation configurations. We varied the simulation time to have a better study.

Table 1. Simulation Parameters.

\begin{tabular}{|l|c|}
\hline \multicolumn{1}{|c|}{ Parameter } & Value \\
\hline Radio Type & 802.16 Radio \\
\hline Antenna Model & Omni directional \\
\hline Channel Frequency & $2.4 \mathrm{GHz}$ \\
\hline Transmission Power & $20 \mathrm{GHz}$ \\
\hline Scheduling Mode & Centralized \\
\hline Traffic Type & CBR \\
\hline Number of IPTV Channel & 50 \\
\hline Precedence Values & $0,2,3,4$ and 7 \\
\hline Scheduling Algorithm Evaluated & SP, WRR, MWRR, AWRR \\
\hline Transport Protocol & UDP \\
\hline
\end{tabular}

\subsection{Simulation Result}

To evaluate and compare the performance of these algorithms; WRR, SP, MWRR, and AWRR, we performed some scenarios adopting conventional metrics. The metrics are average throughput and average end-to-end delay. We studied these metrics either in relation to all service classes or to a specific class, for example rtPS class. We also studied the total number of IPTV channels that scheduler can handle. We present the results of this metric with statistical analysis. 
Figure 2 shows the average throughput compared to the five service classes. Traffic classes of UGS and ertPS have a better throughput performance while using SP scheduler. However, the traffic of rtPS class provides the best performance in terms of throughput with the proposed AWRR algorithm. Indeed, the use of input scheduler has improved the performance of rtPS class favoring encoded IPTV services such as HD and SD. Figure 2 also shows that WRR and MWRR schedulers offer a better result in terms of throughput for low priority classes (nrtPS and BE). Furthermore, the results show that the proposed scheduler has a better performance in terms of average throughput of all classes. This is due to the proposed weight adjustment mechanisms which take into account the QoS constraints and the number of packet occupying each queue.

Figure 3 illustrates the average end-to-end delay of four scheduling algorithms with respect to different service classes. We realize that AWRR algorithm offers the best performance in terms of average end-to-end delay, particularly at rtPS class. These performances could allow achieving an average delay about $13 \%$. Also we can see that for UGS class, SP algorithm has the best delay because the packets with high priority queues are completely served before the low-priority one. Unlike WRR, AWRR gives a good result since it adjusts the weight of the queue for each service class dynamically. Furthermore, the use of input scheduler allowed accelerates packet processing with high-quality video streams.

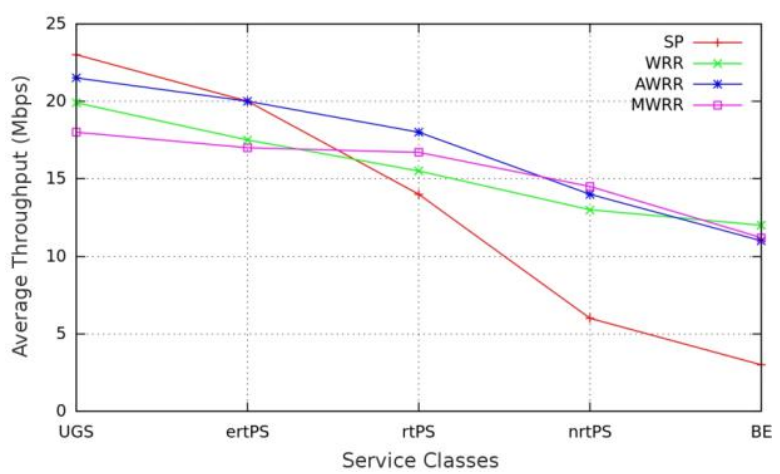

Figure 2. Average throughput over service classes.

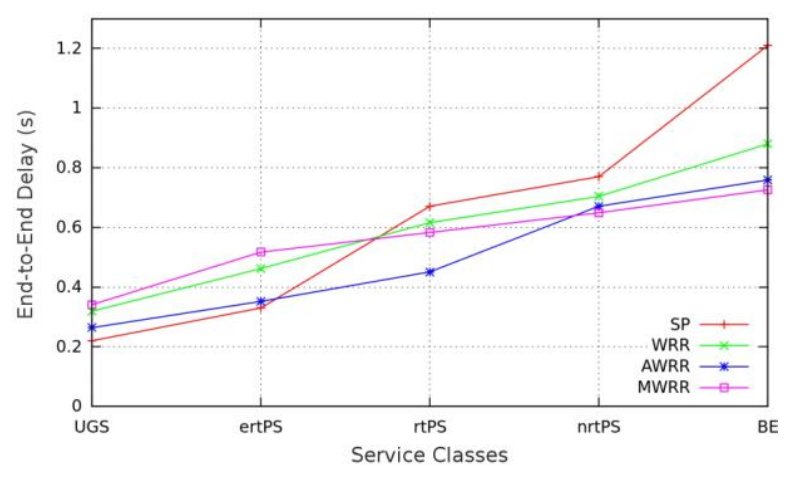

Figure 3. The average of end-to-end delay.

Figures 4 and 5 show the average throughput and the average end-to-end delay of rtPS class respectively. Thus, as we mentioned earlier, the video stream of IPTV services is part of rtPS class. To evaluate the performance of this service class, we changed the number of IPTV channels user-requested. Each channel can be requested with one of qualities (HD-TV, SD-TV, Web-TV and Mobile-TV) in a random manner.

Figures 4 and 5 confirm that the proposed scheduling, AWRR algorithm, provides the best throughput and the best average end-to-end delay. AWRR has average $8 \%$ in terms of throughput and $19 \%$ in terms of end-to-end delay. These results achieved because of the architecture of proposed algorithm using two schedulers which reduce end-to-end delay for video services. Another point about the performance of rtPS class is the fact that generating a lot of traffic at IPTV services class caused exceeding the queue threshold. The reason is that AWRR algorithm triggers the weight adjustment increasing the weight value of rtPS class queue. It becomes one of the advantages of the proposed algorithm that it can handle more 
packets of rtPS class.

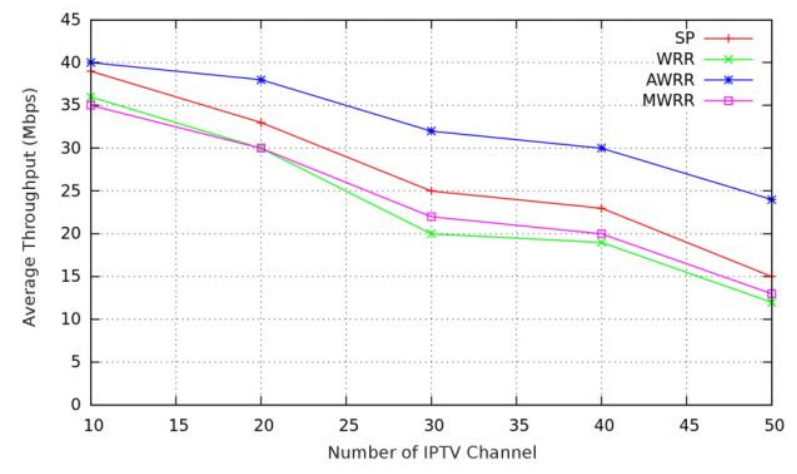

Figure 4. Average throughput of rtPS class.

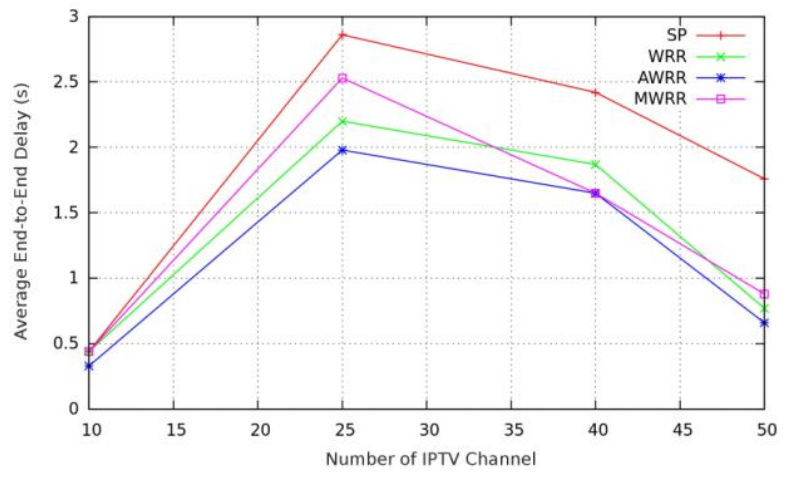

Figure 5. Average end-to-end delay of rtPS class.

In order to better study the behaviour of these four algorithms with low priority classes, we evaluated the performance of nrtPS class by changing the number of CBR connection. Figures 6 and 7 depict the average throughput and the average end-to-end delay of nrtPS class respectively. These figures show that the WRR, AWRR, and MWRR have better result compared to SP algorithm, because it serves UGS, rtPS and ertPS classes before nrtPS class. Moreover, despite a slight superiority of MWRR algorithm, the proposed scheduler offers the best results with its adaptation mechanism.

To highlight the interest of the proposed algorithm, we performed statistical test at the next section. This statistical test allows to compare the performance of WRR, SP, MWRR and

AWRR against several metrics. The result of this comparison can provide which algorithm is the best one in terms of all the metrics for all service classes.

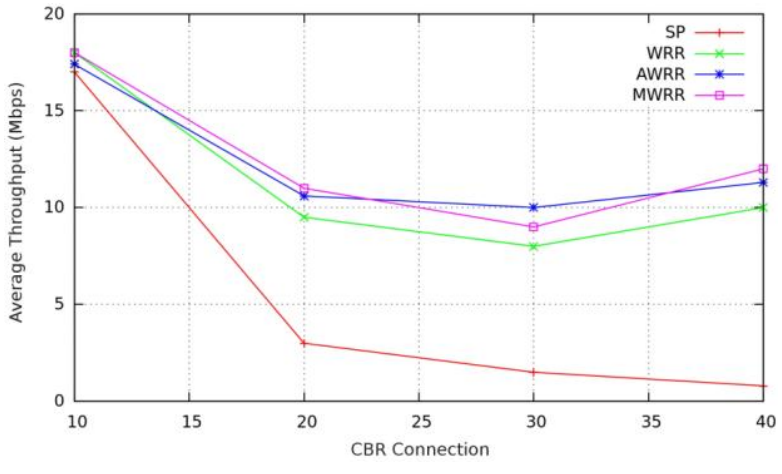

Figure 6. Average throughput of nrtPS class.

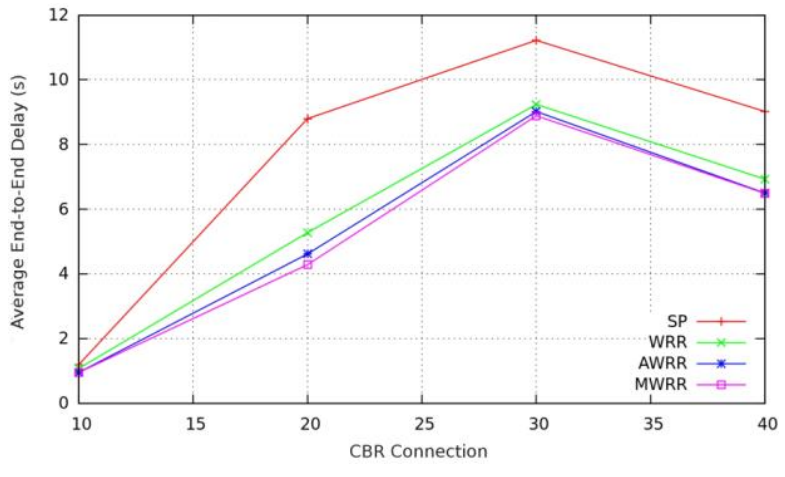

Figure 7. Average end-to-end delay of nrtPS class.

\subsection{Statistical Analysis of Algorithms}

\subsubsection{Statistic Tests}

The performance evaluation of proposed AWRR algorithm demonstrated its efficiency of one service class to another respectively. It could better for one service but not for the others. The goal is to have a global view of performance of proposed AWRR algorithm compared with other algorithms. 
Statistical tests are tools for decision support system. They can decide between one and two cases but only one is true. Before performing the Friedman test, we made some basic definitions as follows:

Definition IV.1. A statistical hypothesis is a statement concerning the characteristics (parameter values, form of observations distribution) of a population.

Definition IV.2. Statistical hypothesis is a fixed assumption that has parameter population priority at a particular value which called null hypothesis, denoted by $H_{0}$. Other assumption that differs from the hypothesis $H_{0}$ called alternative hypothesis and denoted by $H_{1}$.

Definition IV.3. A hypothesis test is a method that aims to provide a decision rule, based-on results sample, to make a choice between two hypotheses statistics.

One of the objectives of a hypothesis test is to predict in advance at what condition that one or other hypothesis will be considered. $H_{0}$ is a subject that being tested and the whole testing process is done by considering this hypothesis as true. If the test leads to the rejection of $H_{0}$, then it considers $H_{1}$ as probable rather than $H_{0}$.

To establish the credibility of $H_{0}$, we should be able to establish decision rules that will succeed without ambiguity to non-rejection of $H_{0}$ (or rejection). In practice, we implement an approach that allows, a long-term, of wrongly rejecting $H_{0}$ true in a small cases proportion. The conclusion is deduced from the sample results following the decision rule.

Definition IV.4. The significance level of a hypothesis test is agreed to advance the risk of wrongly rejecting $H_{0}$ when it is true (and then promote $H_{1}$ ). This level of significance is expressed in probability as follows: $\alpha=$ Prob [reject $H_{0}-H_{0}$ true] $=$ Probability [choose $H_{0}$ $-H_{1}$ true $]$.

At this level of significance, we can be matched on the statistic sampling distribution, a rejection region of $H_{0}$ (also called the critical region). The area of this region corresponds to the probability $\alpha$ (usually set at $5 \%$ or sometimes $1 \%$ ). The region rejection of $H_{0}$ consists of a statistical set values that will lead to rejection of $H_{0}$. For example, if we use a significance level $\alpha=5 \%$, it means that we assume the statistic may take $5 \%$ of cases, a value ranging in the region of rejection of $H_{0}$, although the hypothesis $H_{0}$ is true, and this only after the random sampling. At sampling distribution is also a complementary region, called non-rejection region of $H_{0}$ (also called acceptance region) with probability $1-\alpha$. The observed value of the statistic results derived from the sample belongs, either in the rejection region of $H_{0}$, or in the non-rejection region of $H_{0}$.

\subsubsection{The Friedman Test}

To compare the performance of algorithms AWRR, WRR, SP and MWRR, we performed the Friedman test, knowing that more research interested in this test [12], [13].

The Friedman test is a test for comparing $K$ parameters local. But unlike Kruskal-Wallis, for example, the values are only comparable within each group. This is the differences between the Friedman test and the other statistical tests for independent samples when the 


\section{Macrothink}

ranks built from the all observations measured.

The analysis of variance (ANOVA) of Friedman is to compare $K$ parameters local on $K$ related samples. Therefore the data table has $n$ rows and $K$ columns. The lines represent the interest metric (throughput, delay, jitter, etc.) where the value is expressed by the average or the standard deviation. However, the columns represent the compared algorithms. In its construction, each row corresponds to a " $K$ - tuple" measurements. These can be continuous or ordinal, the essential is we can exploit to produce a classification. It means that it affects to treatment ranks. By assumption, the shapes of $K$ distributions samples are identical, even though they would be offset. In particular they must have the same dispersion [14]. Table 2 shows the samples list that analyzed with the Friedman test.

Here are the two hypotheses statistics of this test:

- $H_{0}$ : there is no difference between the algorithms studied.

- $H_{1}$ : at least one algorithm is different from others.

To perform the Friedman test, first it needs to determine the rank of each algorithm for each sample being studied. Table 3 gives the rank of algorithms based on the average of each sample. $A_{2}$ is the sum of total square ranks:

$$
\begin{gathered}
A_{2}=\sum_{i=1}^{b} \sum_{j=1}^{k}\left[R\left(X_{i j}\right)\right]^{2} \\
A_{2}=21+114+117+108=360
\end{gathered}
$$

and $B_{2}$ is given by the following relationship:

$$
\begin{gathered}
B_{2}=\frac{1}{b} \sum_{j=1}^{k} R_{j}^{2} \\
B_{2}=\frac{1}{12}\left(15^{2}+36^{2}+35^{2}+34^{2}\right)=325.1666
\end{gathered}
$$

Where $b$ is the number of samples studied and $\mathrm{k}$ the number of algorithms to compare.

Then we calculate the statistical test $\mathrm{T}_{2}$ using the following formula:

$$
\begin{gathered}
T_{2}=\frac{(b-1)\left[B_{2}-b k(k+1)^{2} / 4\right.}{A_{2}-B_{2}} \\
T_{2}=7.947
\end{gathered}
$$

Using the distribution table $F$ with $\alpha=1 \%$, we get the following value:

$$
F_{1-\alpha, k-1,(b-1)(k-1)}=F_{0.99,3,33}=4.43678
$$


We chose $\alpha=1 \%$ because most of $\alpha$ is less than the high critical value which makes the strict analysis.

Since $T_{2}>F_{0.99,4,36}$, assuming $H_{0}$ is rejected, i.e there is at least one algorithm whose performance is different from at least one of the other algorithms studied.

To determine which algorithm is different, we performed the test bilateral and results are given in Table 4. Indeed, two algorithms $A$ and $B$ are different if $\left|R_{A}-R_{B}\right|$ is greater than the critical value $C$, where $R$ is the sum of ranks algorithm $i$. The critical value $C$ is calculated using the following expression:

$$
C=t_{1-\frac{\alpha}{2}}\left\lfloor\left.\frac{2 b\left(A_{2}-B_{2}\right)}{(b-1)(k-1)}\right|^{\frac{1}{2}}\right.
$$

Where $t_{1-\frac{\alpha}{2}}$ is the $\left(1-\frac{\alpha}{2}\right)^{\text {th }}$ coefficient of distribution $t$ and $(b-1)(k-1)$ is the degrees number of the system.

$$
C=2.733\left[\frac{2 \times 12 \times(360-325.1666)}{11 \times 3}\right]^{\frac{1}{2}}=13.7558
$$

With $\alpha=3 \%$ and $5 \%$ the critical value $C$ is equal to 11.415 and 10.24 respectively, so that the critical value allows more strict analysis.

Table 4 shows that the results obtained by proposed AWRR algorithm is different from those obtained by the algorithms WRR, SP and MWRR. From this analysis, we can say that proposed AWRR outperforms WRR, SP and MWRR algorithms.

The result obtained by the Friedman test shows that the proposed algorithm (AWRR) provides better performance in a comprehensive manner. 
Table 2. List of samples.

\begin{tabular}{|c|c|c|c|c|c|c|}
\hline StDev & Metric & Avg/ET & AWRR & WRR & SP & MWRR \\
\hline \multirow{2}{*}{$E_{1}$} & \multirow{2}{*}{ Avg. Throughput (all classes) } & Average & 16.5 & 15.58 & 13.2 & 15.48 \\
\hline & & Std Dev. & 3.847077 & 2.892335 & 7.730459 & 2.426026 \\
\hline \multirow{2}{*}{$E_{2}$} & \multirow{2}{*}{ end-to-End Delay (all classes) } & Average & 0.4994 & 0.5962 & 0.6402 & 0.5632 \\
\hline & & Std Dev. & 0.187788 & 0.1936 & 0.350788 & 0.130969 \\
\hline \multirow{2}{*}{$E_{3}$} & \multirow{2}{*}{ Avg. Jitter (all classes) } & Average & 0.0126 & 0.015 & 0.012 & 0.0162 \\
\hline & & Std Dev. & 0.007422 & 0.010916 & 0.005499 & 0.010058 \\
\hline \multirow{2}{*}{$E_{4}$} & \multirow{2}{*}{ Avg. Throughput (rtPS class) } & Average & 32.8 & 23.4 & 27 & 24 \\
\hline & & Std Dev. & 5.74108 & 8.52291 & 8.294577 & 7.720104 \\
\hline \multirow{2}{*}{$E_{5}$} & \multirow{2}{*}{ End-to-End Delay (rtPS class) } & Average & 5.275 & 5.6325 & 7.56 & 5.1575 \\
\hline & & Std Dev. & 2.939979 & 2.981068 & 3.791912 & 2.918847 \\
\hline \multirow{2}{*}{$E_{6}$} & \multirow{2}{*}{ Avg. Throughput (nrtPS class) } & Average & 12.325 & 11.375 & 5.575 & 12.5 \\
\hline & & Std Dev. & 2.965953 & 3.895109 & 6.643935 & 3.354102 \\
\hline \multirow{2}{*}{$E_{7}$} & \multirow{2}{*}{$\begin{array}{l}\text { End-to-End Delay (nrtPS } \\
\text { class) }\end{array}$} & Average & 1.155 & 1.32 & 1.87 & 1.375 \\
\hline & & Std Dev. & 0.680312 & 0.733792 & 0.913729 & 0.795126 \\
\hline \multirow{2}{*}{$E_{8}$} & \multirow{2}{*}{ Packet loss } & Average & 0.096333 & 0.099 & 0.097533 & 0.102667 \\
\hline & & Std Dev. & 0.021312 & 0.041158 & 0.040019 & 0.034003 \\
\hline \multirow{2}{*}{$E_{9}$} & \multirow{2}{*}{ Packet loss (Traffic overload) } & Average & 62.33333 & 65.33333 & 63 & 65.66667 \\
\hline & & Std Dev. & 8.806563 & 11.11555 & 8.164966 & 10.65624 \\
\hline \multirow{2}{*}{$E_{10}$} & \multirow{2}{*}{$\begin{array}{l}\text { Number of IPTV channels } \\
\text { sent }\end{array}$} & Average & 4.925 & 3.625 & 4.25 & 3.975 \\
\hline & & Std Dev. & 1.851182 & 1.810214 & 1.916377 & 1.884642 \\
\hline \multirow{2}{*}{$E_{11}$} & \multirow{2}{*}{$\begin{array}{l}\text { Number of IPTV channels } \\
\text { (HD/SD) }\end{array}$} & Average & 3.65 & 2.25 & 2.7 & 2.55 \\
\hline & & Std Dev. & 1.930673 & 1.752855 & 2.043282 & 1.908533 \\
\hline \multirow{2}{*}{$E_{12}$} & \multirow{2}{*}{$\begin{array}{l}\text { Number of IPTV channels } \\
\text { (Web/Mobile) }\end{array}$} & Average & 1.475 & 1.325 & 1.05 & 1.225 \\
\hline & & Std Dev. & 0.588961 & 0.641775 & 0.606218 & 0.605702 \\
\hline
\end{tabular}

Table 3. Algorithms Classification.

\begin{tabular}{|c|c|c|c|c|c|c|c|c|c|c|c|c|}
\hline & \multicolumn{3}{|c|}{ AWRR } & \multicolumn{3}{|c|}{ WRR } & \multicolumn{3}{|c|}{ SP } & \multicolumn{3}{|c|}{ MWRR } \\
\hline Instances(b) & $X_{b 1}$ & $R\left(X_{b 1}\right)$ & $R\left(X_{b 1}\right)^{2}$ & $X_{b 2}$ & $R\left(X_{b 2}\right)$ & $R\left(X_{b 2}\right)^{2}$ & $X_{b 3}$ & $R\left(X_{b 3}\right)$ & $R\left(X_{b 3}\right)^{2}$ & $X_{b 4}$ & $R\left(X_{b 4}\right)$ & $R\left(X_{b 4}\right)^{2}$ \\
\hline$E_{2}$ & 0.4994 & 1 & 1 & 0.5962 & 3 & 9 & 0.6402 & 4 & 16 & 0.5632 & 2 & 4 \\
\hline$E_{4}$ & -32.8 & 1 & 1 & -23.4 & 4 & 16 & -27.0 & 2 & 4 & -24.0 & 3 & 9 \\
\hline$E_{5}$ & 5.275 & 2 & 4 & 5.6325 & 3 & 9 & 7.56 & 4 & 16 & 5.1575 & 1 & 1 \\
\hline$E_{6}$ & -12.325 & 2 & 4 & -11.375 & 3 & 9 & -5.575 & 4 & 16 & -12.5 & 1 & 1 \\
\hline$E_{8}$ & 0.09633 & 1 & 1 & 0.099 & 3 & 9 & 0.09753 & 2 & 4 & 0.10267 & 4 & 16 \\
\hline$E_{8}$ & 62.33333 & 1 & 1 & 65.33333 & 3 & 9 & 63.0 & 2 & 4 & 65.66667 & 4 & 16 \\
\hline$E_{10}$ & -4.925 & 1 & 1 & -3.625 & 4 & 16 & -4.25 & 2 & 4 & -3.975 & 3 & 9 \\
\hline$E_{11}$ & -3.65 & 1 & 1 & -2.25 & 4 & 16 & -2.7 & 2 & 4 & -2.550 & 3 & 9 \\
\hline$E_{12}$ & -1.475 & 1 & 1 & -1.325 & 2 & 4 & -1.05 & 4 & 16 & -1.225 & 3 & 9 \\
\hline Average rank & & 1.25 & & & 3 & & & 2.916 & & & 2.833 & \\
\hline
\end{tabular}


Table 4. Bilateral Test.

\begin{tabular}{|c|c|c|c|}
\hline & WRR & SP & MWRR \\
\hline AWRR & $\mathbf{2 1}$ & $\mathbf{2 0}$ & $\mathbf{1 9}$ \\
\hline WRR & - & 1 & 2 \\
\hline SP & - & - & 1 \\
\hline
\end{tabular}

\section{Conclusion}

We present a scheduling mechanism for video in WiMAX networks. The proposed solution, called Adaptive Weighted Round Robin (AWRR), is designed to optimize the performance of video streams that have more constraints. This technique offers different service in defining priorities and promoting streams belonging to multimedia or real time applications. This differentiation is due to that some applications are generally sensitive to certain parameters such as delay, bandwidth and packet loss rate.

The proposed algorithm is based on two schedulers, input scheduler and output scheduler. The input scheduler uses two queues, a high priority queue for service classes such as UGS, ertPS and rtPS, in case of HD or SD flow, and low priority queue for service classes such as nrtPS, BE and Web-TV or Mobile-TV video streams. The next contribution of proposed solution is the weight adjustment system. In this system, we represent the weight of each queue in form of the first degree equation. We also set a threshold for each queue. These thresholds are used to trigger the weight adjustment. This technique allows to adjust the weights which take into account the QoS constraints of each queue dynamically.

We performed simulation which compares the proposed algorithm with WRR, SP and MWRR schedulers. We also performed a statistical analysis of four schedulers with the Friedman test. The results obtained show that the proposed scheduler has a better performance especially for rtPS service class (multimedia applications). It shows that the proposed AWRR algorithm provides advantages such as a better QoS management, reducing loss of packets and increasing the overall network performance.

In future work, we are particularly interested in studying the possibility of applying the proposed mechanisms (scheduling, path selection and admission control) in conjunction which the main objective is to study the performance of the QoS chain management entire in WiMAX relay networks. Furthermore, we will adapt the proposed solution into HSDPA / LTE technology.

\section{References}

[1] IEEE-Standard. 802.16j-2009 Part 16: Air Interface for Fixed and Mobile Broadband Wireless Access Systems, Multihop Relay Specification. IEEE Standard for Local and Metropolitan Area Networks, 2009.

[2] Francis E Retnasothie, M Kemal Ozdemir, Tevfik Yucek, Hasari Celebi, Joseph Zhang, and Ranesh Muththaiah, "Wireless IPTV over WiMAX: Challenges and Applications", In 
Proceedings of the IEEE Annual Wireless and Microwave Technology Conference, Vol.45 of WAMICON'06, pp. 1-5, Clearwater Beach, FL, December 2006. http://dx.doi.org/10.1109/WAMICON.2006.351905

[3] S Y Wang, H Y Chen, and S W Chuang, "Nctuns Tool for IEEE 802.16j Mobile WiMAX Relay Network Simulations", In Nova Science Publishers, editor, Computer Science Research and Technology, No.1, pp. 1-31. Addison Wesley, 2009.

[4] Aymen Belghith and Loutfi Nuaymi, "Comparison of WiMAX Scheduling Algorithms and Proposals for the RTPS QoS Class", In Proceedings of the 14th European Wireless Conference 2008, pp. 1-6, Prague, Czech Republic, 2008. http://dx.doi.org/10.1109/EW.2008.4623857

[5] Ahtsham Sarwar Khan, Ahsan Razza Sattar, Tasleem Mustafa, and Shehbaz Ahmad, "Performance Evaluation and Enhancement of Uplink Scheduling Algorithms in Point to Multipoint WiMAX Networks", In European Journal of Scientific Research, Vol.42, pp. 491-506. EuroJournals, 2010.

[6] Wail Mardini and Mai Abu Alfool, "Modified WRR Scheduling Algorithm for WIMAX Networks", In Journal of Network Protocols and Algorithms, Vol.3, pp. 24-43, USA, 2011. http://dx.doi.org/10.5296/npa.v3i2.751

[7] Ahmed H. Rashwan, Hesham M. El-Badawy, and Hazem H. Ali, "Comparative Assessments for Different WiMAX Scheduling Algorithms", In Proceedings of the World Congress on Engineering and Computer Science, San Francisco, USA, October 2009.

[8] Ji-Young Kwak, Ji-Seung Nam, and Doo-Hyun Kim, "A Modified Dynamic Weighted Round Robin Cell Scheduling Algorithm”, In ETRI Journal, Vol.24, pp. 360-372, October 2002. http://dx.doi.org/10.4218/etrij.02.0102.0504

[9] Mohamed El Amine Brahmia, Abdelhafid Abouaissa, and Pascal Lorenz, "Adaptive Scheduling Mechanism for IPTV over WiMAX IEEE 802.16j Networks", International Journal of Communication Systems, Vol.27, Issue 7, pp. 1009-1019, 2012. http://dx.doi.org/10.1002/dac.2392

[10]Najah Abu Ali, Pratik Dhrona, and Hossam Hassanein, “A Performance Study of Uplink Scheduling Algorithms in Point-to-Multipoint WiMAX Networks", In Comput. Commun., Vol.32, pp. 511-521, Newton, MA, USA, Butterworth-Heinemann, February 2009. http://dx.doi.org/10.1016/j.comcom.2008.09.015

[11]Scalable Network Technologies (SNT), Qualnet. http://www.scalable-networks.com/content/products/qualnet/.

[12] Salvador Garcia, Daniel Molina, Manuel Lozano, and Francisco Herrera, "A Study on The Use of Non-parametric Tests for Analyzing The Evolutionary Algorithms' Behaviour: A Case Study on The CEC'2005 Special Session on Real Parameter Optimization", Journal of Heuristics, 15(6):617-644, December 2009. http://dx.doi.org/10.1007/s10732-008-9080-4 [13]J.G. Villegas, "Using Nonparametric Test to Compare The Performance of Metaheuristics", http://juangvillegas.files.wordpress.com/2011/08/friedman-test-24062011.pdf, 2011.

[14]Ricco Rakotomalala, "Comparison of Populations, Nonparametric Tests", ttp://eric.univlyon2.fr/ ricco/cours/cours/Comp_Pop_Tests_Nonparametriques.pdf, 2008.

[15]M. Friedman, "The Use of Ranks to Avoid the Assumption of Normality Implicit in the 
Analysis of Variance", Journal of the American Statistical Association, Vol. 32, No.200, pp. 675-701, 1937. http://dx.doi.org/10.1080/01621459.1937.10503522

[16]L. Idoumghar, N. Cherin, R. Roche, P. Siary, A. Miraoui, "Hybrid ICA-PSO Algorithm for Continuous Optimization. Journal of Applied Mathematics and Computation", Vol. 219, No. 24, pp. 11149-11170, 15 August 2013. http://dx.doi.org/10.1016/j.amc.2013.05.027

[17] Abdusy Syarif, Abdelhafid Abouaissa, Lhassane Idoumghar, Riri Fitri Sari and Pascal Lorenz, "Performance Analysis of Evolutionary Multi-Objective Based Approach for Deployment of Wireless Sensor Network with The Presence of Fixed Obstacles", IEEE Global Communication Conference (Globecom), Austin, Texas, USA, 8-12 December, 2014. [18]Luigi Boschetti, Annamaria Kunzle, Pietro Alessandro Brivio, Luigi Mussio, "Nonparametric Statistical Tests for The Analysis of Multiple-Sensor Time Series of Remotely Sensed Data", IEEE International Conference on Geoscience and Remote Sensing Symposium (IGARSS),pp. 200-203, $31 \quad$ July-4 Aug, 2006. http://dx.doi.org/10.1109/IGARSS.2006.56

[19]Ming Jin, Youming Li, Gang Wang, Jiehui Chen, "Multiantenna Based Spectrum Sensing via Friedman Test for Cognitive Radio", 7th International ICST Conference on Communications and Networking in China (CHINACOM), pp. 321-324, 2012. http://doi.ieeecomputersociety.org/10.1109/ChinaCom.2012.6417499

[20]Tetsuya Oda, Admir Barolli, Evjola Spaho, Leonard Barolli and Fatos Xhafa, "Analysis of Mesh Router Placement in Wireless Mesh Networks Using Friedman Test", IEEE 28th International Conference on Advanced Information Networking and Applications, pp. 289-296, 2014. http://dx.doi.org/10.1109/AINA.2014.152

[21]Liang Zhou, Zhen Yang, Yonggang Wen, Joel J. P. C. Rodrigues, "Distributed Wireless Video Scheduling with Delayed Control Information", IEEE Transactions on Circuits and Systems for Video Technology, IEEE, ISSN: 1051-8215, Vol. 24, No. 5, May 2014, pp. 889-901, http://dx.doi.org/10.1109/TCSVT.2013.2291311

[22]Jaime Lloret, Alejandro Canovas, Joel J. P. C. Rodrigues, Kai Lin, "A Network Algorithm for 3D/2D IPTV Distribution using WiMAX and WLAN Technologies", in Multimedia Tools and Applications, Springer, Vol. 67, Issue 1, November 2013, pp. 7-30, DOI: 10.1007/s11042-011-0929-4.

[23]João Rodrigues, António Nogueira, Paulo Salvador, Joel J. P. C. Rodrigues, "IPTV Service based on a Content-Zapping Paradigm", in Multimedia Systems Journal, Springer, Volume 17, Issue 4, 2011, pp. 351-364. DOI: 10.1007/s00530-010-0215-8

\section{Copyright Disclaimer}

Copyright reserved by the author(s).

This article is an open-access article distributed under the terms and conditions of the Creative Commons Attribution license (http://creativecommons.org/licenses/by/3.0/). 